\title{
Pertussis toxin blocks growth factor receptor signalling by attenuating $\mathrm{p} 2$ I ras activity DA Eisinger* and $\mathrm{H}$ Ammer
}

Address: Institute of Pharmacology, Toxicology and Pharmacy, München, Germany

* Corresponding author

from 12th Joint Meeting of the Signal Transduction Society (STS). Signal Transduction: Receptors, Mediators and Genes Weimar, Germany. 29-3I October 2008

Published: 26 February 2009

Cell Communication and Signaling 2009, 7(Suppl I):A44 doi:I0.II86/I478-8IIX-7-SI-A44

This abstract is available from: http://www.biosignaling.com/content/7/SI/A44

C 2009 Eisinger and Ammer; licensee BioMed Central Ltd.

Pertussis toxin (PTX), a Bordetella Pertussis exotoxin, has been recently shown to prevent growth factor-induced activation of the extracellular signal regulated protein kinases ERK1 and ERK2. Due to its capacity to ribosylate inhibitory GTP-binding proteins of the Gi/o family, the antiproliferative effect of PTX is generally thought to originate form interference with growth factor receptorinduced inhibitory signal transduction pathways. However, in human embryonic kidney (HEK293) cells, we could recently demonstrate that attenuation of inhibitory Gi/o signaling by the regulator protein Goloco failed to affect epidermal growth factor (EGF) receptor-induced ERK1/2 activation. In addition, Goloco also failed to interfere insulin-like growth factor (IGF-1) receptor associated ERK1/2 signaling, indicating that PTX must affect ERK1/2 signalling by mechanism other than inactivation of Gi/o function. The small GTP-binding protein p21ras plays a central role in mitogenic signalling, as it connects a number of growth factor receptors to the raf-1/MEK/ ERK1/2 signalling module. Western blot experiments revealed that pre-treatment of HEK293 cells with PTX prevents translocation of p21ras to the plasma membranes. Moreover, immune-precipitation experiments also showed that PTX prevents interaction of p21ras with raf-1 kinase. A similar finding was observed after pre-treatment of the cells with mevastatin, a 3-hydroxy-3-methylglutaryl coenzyme A reductase inhibitor. Mevastatin treatment resulted in a loss of p21ras from the plasma membrane, inhibited EGF-induced p21ras/raf-1 interaction and ERK1/2 activation. These results indicate that, besides of its inhibitory action on Gi/o signalling pathways, PTX may also interfere with growth factor-mediated ERK1/2 activation by attenuating p21ras activation. 